Fonoaudiologia Baseada

em Evidências

Evidence-based Speech-Language

Pathology and Audiology

Helena Perrut Veiga ${ }^{1}$

Esther Mandelbaum Gonçalves Bianchini'

Descritores

Comportamento de ingestão de líquido Adulto

Idoso

Deglutição

Revisão

Keywords

Drinking behavior

Adult

Aged

Deglutition

Review

Endereço para correspondência:

R. Cb. Geraldo Calderaro, 75, bl.2/303,

Jacarepaguá, Rio de Janeiro (RJ), Brasil, CEP: 22770-290.

E-mail: helenaperrut@hotmail.com

Recebido em: 12/4/2012

Aceito em: 6/8/2012

\section{Características da deglutição sequencial de líquidos em adultos e idosos: uma revisão integrativa}

\section{Characteristics of sequential swallowing of liquids in young and elderly adults: an integrative review}

Objetivo: Realizar revisão integrativa de estudos sobre deglutição sequencial de líquidos, caracterizando o método e os principais achados em adultos e idosos. Estratégia de pesquisa: Foi realizado levantamento na literatura internacional, publicada nos idiomas Inglês ou Português, utilizando-se base de dados PubMed, LILACS, SciELO, MEDLINE, dos últimos 20 anos, cujos artigos estavam disponíveis na íntegra. Foram utilizadas as seguintes palavras, em combinações variadas: deglutição sequencial, deglutição, disfagia, copo e canudo. Critérios de seleção: Foram incluídos artigos de pesquisa com abordagem metodológica referente à caracterização da deglutição sequencial de líquidos em sujeitos adultos e/ou idosos, independentemente de patologia envolvida. Excluíram-se os estudos que englobavam apenas a fase esofágica. Análise dos dados: Foram considerados indicadores da pesquisa: objetivos, número e gênero dos participantes; faixa etária; quantidade de líquido ofertado; instrução para ingesta; utensílio e métodos utilizados; principais resultados. Resultados: Dezoito estudos contemplaram os critérios propostos. Os artigos foram tabulados quanto à caracterização da amostra e à metodologia (volume ofertado, utensílio utilizado e tipos de exames). A maioria dos artigos envolveu apenas indivíduos saudáveis e sem queixas de deglutição. Houve diferentes tipos de instrução para deglutir todo o volume: maneira habitual; continuamente; o mais rápido possível. Os achados referentes às caracterizações da deglutição sequencial foram variados e descritos de acordo com objetivos de cada estudo. Conclusão: Constata-se grande variabilidade na metodologia empregada para caracterização da deglutição sequencial. Alguns achados não são comparáveis entre si, sendo que a deglutição sequencial ainda não faz parte da maioria dos protocolos de avaliação da deglutição. Não há consenso sobre a influência do utensílio.

\begin{abstract}
Purpose: To perform an integrative review of studies on liquid sequential swallowing, by characterizing the methodology of the studies and the most important findings in young and elderly adults. Research strategy: Review of the literature written in English and Portuguese on PubMed, LILACS, SciELO and MEDLINE databases, within the past twenty years, available fully, using the following uniterms: sequential swallowing, swallowing, dysphagia, cup, straw, in various combinations. Selection criteria: Research articles with a methodological approach on the characterization of liquid sequential swallowing by young and/or elderly adults, regardless of health condition, excluding studies involving only the esophageal phase. Data analysis: The following research indicators were applied: objectives, number and gender of participants; age group; amount of liquid offered; intake instruction; utensil used, methods and main findings. Results: 18 studies met the established criteria. The articles were categorized according to the sample characterization and the methodology on volume intake, utensil used and types of exams. Most studies investigated only healthy individuals, with no swallowing complaints. Subjects were given different instructions as to the intake of all the volume: usual manner, continually, as rapidly as possible. The findings about the characterization of sequential swallowing were varied and described in accordance with the objectives of each study. Conclusion: It found great variability in the methodology employed to characterize the sequential swallowing. Some findings are not comparable, and sequential swallowing is not studied in most swallowing protocols, without consensus on the influence of the utensil.
\end{abstract}

Trabalho realizado no Programa de Mestrado Profissional em Fonoaudiologia, Universidade Veiga de Almeida - UVA - Rio de Janeiro (RJ), Brasil.

(1) Programa de Pós-Graduação (Mestrado Profissional) em Fonoaudiologia, Universidade Veiga de Almeida - UVA - Rio de Janeiro (RJ), Brasil.

Conflito de interesses: Não 


\section{INTRODUÇÃO}

O modo de deglutir líquidos apresenta variação conforme o volume apresentado, permitindo classificar a deglutição como única ou sequencial. A deglutição única ou isolada acontece quando um pequeno volume é deglutido de uma só vez. Entretanto, para esse mesmo volume, podem ocorrer deglutições múltiplas, principalmente nos sujeitos disfágicos ${ }^{(1)}$, sendo essas ocorrências amplamente investigadas nos protocolos de avaliação da deglutição, tanto clínicos quanto instrumentais ${ }^{(1-3)}$.

A deglutição sequencial, também chamada de contínua( ${ }^{(4)}$ ou consecutiva ${ }^{(5)}$, ocorre quando há múltiplas e consecutivas deglutições de um grande volume ${ }^{(6,7)}$, com pausas ou sem pausas, com interrupções da respiração( ${ }^{(8)}$. A avaliação da deglutição sequencial fornece ao clínico informações acerca do fluxo do bolo, que são relevantes para o típico comportamento de ingesta realizado fora de um ambiente radiológico bem estruturado e controlado $^{(9)}$. Ela permite, com isso, uma tomada de decisão mais acertada quanto aos parâmetros para liberação de dieta por via oral.

A análise da deglutição sequencial tem merecido cada vez mais atenção por se assemelhar ao modo natural de deglutir líquidos $^{(9,10)}$, onde cada sujeito regula a quantidade ingerida ${ }^{(7)}$, e por apresentar padrões diferenciados da deglutição isolada ${ }^{(11)}$. Além disso, estudos mostram que são necessárias de cinco a sete deglutições por tipo de bolo para obtenção de um grau de confiança nas medidas da fase faríngea ${ }^{(12)}$.

Pesquisas mostram que o utensílio e o tamanho do copo influenciam no volume ingerido ${ }^{(13)}$. Portanto também devemos observar com quais utensílios os diferentes estudos têm sido conduzidos.

Volumes maiores de líquido têm sido utilizados principalmente nos testes clínicos de triagem para risco de disfunção da deglutição ${ }^{(14)}$, realizados geralmente por outros profissionais de saúde. Em geral, não há padronização no volume e no modo de oferta desses líquidos ${ }^{(6,15)}$.

Para melhor esclarecer e identificar as principais características da deglutição sequencial, torna-se necessário coletar e analisar trabalhos que abordem esse tema, buscando verificar as evidências disponíveis na literatura.

\section{OBJETIVO}

Realizar uma revisão integrativa sobre a produção científica referente à deglutição sequencial de líquidos nas duas últimas décadas, caracterizando o método utilizado para coleta de dados e os principais achados, em adultos e idosos com ou sem queixa de deglutição.

\section{ESTRATÉGIA DE PESQUISA}

Para o alcance do objetivo proposto, foi realizada uma revisão integrativa da literatura, com a delimitação das seguintes etapas $^{(16)}$ : identificação do problema com elaboração da pergunta norteadora; estabelecimento de palavras-chave e de critérios para inclusão/exclusão de artigos; seleção dos artigos; definição das informações a serem extraídas dos trabalhos revisados, tais como objetivos, metodologia e principais conclusões.

A pergunta que norteou o presente estudo foi: "Como se caracteriza a deglutição sequencial de líquidos em adultos e idosos?". Para seleção dos artigos, houve levantamento na literatura internacional, publicada nos idiomas Inglês ou Português, utilizando-se base de dados PubMed, LILACS, SciELO e MEDLINE. Foram incluídos artigos publicados nos últimos vinte anos (1991 e 2011), e que estivessem disponíveis na íntegra. Foram utilizadas as seguintes palavras, em combinações variadas: deglutição sequencial, deglutição, disfagia, copo e canudo. Para a busca em Inglês foram utilizadas as palavras equivalentes (sequential swallowing, deglutition, dysphagia, cup, straw), também em combinações variadas.

As referências bibliográficas dos artigos selecionados foram analisadas para verificar outros estudos que pudessem ter sido omitidos, durante a busca eletrônica inicial.

A partir dessas estratégias de pesquisa, foram encontrados 24 artigos. As análises foram feitas inicialmente quanto aos critérios de seleção descritos a seguir.

\section{Critérios de seleção}

Foram incluídos artigos cuja abordagem metodológica fosse referente à deglutição sequencial de líquidos com amostra constituída por sujeitos adultos e/ou idosos, independentemente da possível patologia envolvida, uma vez que se objetivou caracterizar o estudo da deglutição sequencial nessa faixa etária. Embora a inclusão de pesquisas que envolvam não somente sujeitos saudáveis traga maior número de variáveis a esse estudo, optou-se por mantê-los, visto a quantidade restrita dos estudos enfocando a deglutição sequencial.

Numa segunda etapa, seis artigos foram excluídos por não irem ao encontro dos objetivos desta revisão integrativa: estudos cuja metodologia não incluiu explicitamente deglutições sequenciais ${ }^{(17)}$, mesmo que se referissem a volumes maiores de líquido; estudos que utilizaram a deglutição sequencial como um método de triagem, utilizado pelo fonoaudiólogo ou por outros profissionais de saúde ${ }^{(8,14,18-20)}$.

Foram excluídas as pesquisas realizadas: exclusivamente com crianças; exclusivamente com a fase esofágica da deglutição; com deglutição sequencial apenas de pastosos ou sólidos. Citações que não permitiram o acesso ao texto na íntegra ou que fossem em outras línguas que não o Inglês ou o Português também foram excluídas.

\section{Análise dos dados}

A análise dos dados foi realizada de forma descritiva, em duas etapas. Primeiramente, foram considerados os seguintes dados: objetivos; número e gênero dos participantes; faixa etária; quantidade de líquido e alimento ofertado; instrução dada para a ingesta; utensílio utilizado; método que avaliou a deglutição; e principais resultados do estudo.

$\mathrm{Na}$ segunda etapa, os estudos foram agrupados e analisados de acordo com os achados das fases oral e faríngea da deglutição. A escolha de tais variáveis baseou-se na possibilidade de aglutinação de informações de interesse quanto à caracterização 
da deglutição sequencial, assim como na devida verificação dos estudos quanto à caracterização da amostra.

\section{RESULTADOS}

Foram encontrados 18 estudos que contemplaram os critérios de inclusão e de exclusão, no período analisado, todos em Inglês. Obtiveram-se os resultados quanto à caracterização da amostra e quanto à divisão ou não por faixa etária e gênero (Quadro 1). Três dos estudos desta revisão, conduzidos por um mesmo grupo ${ }^{(4,21,22)}$, consideram como idosos os indivíduos com idade superior a 50 anos.
Dos 18 estudos analisados, o número de participantes variou de 5 a 345, com média de 40,6 e mediana de 17,5 participantes. A mediana foi considerada a medida mais adequada para mostrar essa distribuição.

Os resultados quanto à metodologia, volume ofertado, utensílio utilizado e tipos de exames realizados também foram obtidos (Quadro 2). Os estudos foram elencados em ordem cronológica de apresentação, com a numeração correspondente às referências bibliográficas, possibilitando visualizar a evolução de como a deglutição sequencial vem sendo estudada.

Do total de artigos analisados, $83,3 \%(\mathrm{n}=15)$ dos artigos estudaram apenas indivíduos saudáveis e sem queixas de

Quadro 1. Caracterização da amostra quanto à divisão ou não por faixa etária e gênero

\begin{tabular}{|c|c|c|c|c|c|c|}
\hline & \multicolumn{2}{|c|}{ Dividiu variáveis } & \multicolumn{2}{|c|}{ Não dividiu variáveis } & \multicolumn{2}{|c|}{ Abordou apenas um tipo de variáve } \\
\hline & $\begin{array}{l}\text { Total de } \\
\text { estudos }\end{array}$ & $\begin{array}{c}\text { Números dos estudos } \\
\text { citados* }^{*}\end{array}$ & $\begin{array}{l}\text { Total de } \\
\text { estudos }\end{array}$ & $\begin{array}{c}\text { Números dos estudos } \\
\text { citados* }\end{array}$ & $\begin{array}{l}\text { Total de } \\
\text { estudos }\end{array}$ & $\begin{array}{c}\text { Números dos estudos } \\
\text { citados* }\end{array}$ \\
\hline $\begin{array}{l}\text { Idade (adultos e } \\
\text { idosos) }\end{array}$ & 6 & $4 ; 11 ; 21 ; 22 ; 32 ; 33$ & 3 & $9 ; 12 ; 26$ & 9 & $\begin{array}{c}5 ; 23 ; 24 ; 25 ; 27 \\
28 ; 29 ; 30 ; 31\end{array}$ \\
\hline Gênero & 14 & $\begin{array}{c}4 ; 21 ; 22 ; 23 ; 24 ; \\
25 ; 26 ; 27 ; 28 ; 29 \\
30 ; 31 ; 32 ; 33\end{array}$ & 2 & $9 ; 12$ & 2 & $5 ; 11$ \\
\hline
\end{tabular}

*Utilizou-se o número do respectivo estudo na referência

Quadro 2. Metodologia dos estudos analisados, apresentados em ordem cronológica, quanto ao volume ofertado, tipo de utensílio utilizado e de exame realizados

\begin{tabular}{|c|c|c|}
\hline Autores & Volume e utensílio utilizado & Exames utilizados \\
\hline Lazarus et al..$^{(12)}$ & $\begin{array}{l}\text { 1, } 3 \text { e } 5 \mathrm{ml} \text { de liquido }-4 \text { deglutições de cada e } 1 \mathrm{ml} \text { pastoso } \\
-3 \text { deglutições }\end{array}$ & Videofluoroscopia (VFC) \\
\hline Martin et al. ${ }^{(24)}$ & $3,10,20 \mathrm{ml}$ (seringa) $+100 \mathrm{ml}$ (canudo) & $\begin{array}{l}\text { Respirógrafo +Eletromiografia de superfície } \\
(E M G)+\text { Videoendoscopia da deglutição (VED) }\end{array}$ \\
\hline Nilsson et al. ${ }^{.(32)}$ & $200 \mathrm{ml}$ (canudo) & $\begin{array}{l}\text { Sistema Doppler; detector de pressão de } \\
\text { sucção; sensor piezo-elétrico e termodetector } \\
\text { de respiração }\end{array}$ \\
\hline Nilsson et al. ${ }^{(33)}$ & $200 \mathrm{ml}$ (canudo) & $\begin{array}{l}\text { Sistema Doppler; detector de pressão de } \\
\text { sucção; sensor piezo-elétrico e termodetector } \\
\text { de respiração }\end{array}$ \\
\hline Preiksaitis \& Mills ${ }^{(29)}$ & $\begin{array}{l}5 \text { e } 20 \mathrm{ml} \text { (seringa) } \\
200 \mathrm{ml} \text { (copo e canudo) }(2 \mathrm{x})\end{array}$ & Pletismografia + EMG + microfone \\
\hline Dua et al. ${ }^{(27)}$ & $\begin{array}{l}\text { Sólido e líquido - refeição fast-food (G1) } \\
\text { Líquido e sólido em pequena quantidade, com bário (G2) }\end{array}$ & $\begin{array}{l}\text { VED+Respirógrafo+EMG (G1) } \\
\text { VFC+VED+EMG (G2) }\end{array}$ \\
\hline Chi-Fishman et al. ${ }^{(23)}$ & 5,30 (seringa) e $200 \mathrm{ml}$ (copo) (2x) & Eletropalatografia e Ultrassonografia \\
\hline Chi-Fishman \& Sonies ${ }^{(28)}$ & 5,10 (seringa) e $150 \mathrm{ml}$ (copo) (2x) & VFC +EMG \\
\hline Daniels \& Foundas ${ }^{(5)}$ & $300 \mathrm{ml}$ (canudo) em 10seg (2x) & VFC \\
\hline Hirst et al. ${ }^{(30)}$ & $\begin{array}{l}10 \times 5 \mathrm{ml} ; 20 \mathrm{ml} \\
100 \mathrm{ml} \text { (copo e canudo) }\end{array}$ & $\begin{array}{l}\text { Manometria faringoesofágica, oxímetro de pulso } \\
\text { e espirometria }\end{array}$ \\
\hline Steele \& Van Lieshout ${ }^{(4)}$ & $\begin{array}{l}\text { Ensaios com } 8 \text { deglutições sequenciais com } 3 \text { consistências } \\
\text { líquidas (copo) }\end{array}$ & Articulografia magnética \\
\hline Daniels et al..$^{(11)}$ & $300 \mathrm{ml}$ (canudo) em 10seg (2x) & VFC \\
\hline Dozier et al. ${ }^{(26)}$ & $50 \mathrm{ml}(\mathrm{copo})$ & VFC + Fluxômetro nasal \\
\hline Steele \& Van Lieshout ${ }^{(22)}$ & Comparação entre deglutições únicas e sequenciais (copo) & Articulografia magnética \\
\hline Murguia et al..$^{(9)}$ & $100 \mathrm{ml}$ (соро) & VFC \\
\hline Steele \& Van Lieshout ${ }^{(21)}$ & 6 goles de cada tipo, sem controle do volume (copo) & Articulografia magnética \\
\hline Tsushima et al. ${ }^{(25)}$ & $200 \mathrm{ml}$ (canudo) & VFC \\
\hline Lederle et al..$^{(31)}$ & $20 \mathrm{ml}, 88,7 \mathrm{ml}$ e $177,4 \mathrm{ml}$ (3 ensaios de cada) (canudo) & $\begin{array}{l}\text { Magnetômetro respiratório; EMG; oxímetro de } \\
\text { pulso }\end{array}$ \\
\hline
\end{tabular}


deglutição; $16,7 \%$ (n=3) estudaram indivíduos tanto saudáveis quanto com queixas de deglutição.

Quanto à instrução dada para deglutir todo o volume, podemos dividir os estudos em três grandes grupos: $39 \%(n=7)$ orientaram a ingerir da maneira habitual; 27,8\% $(\mathrm{n}=5)$ orientaram a ingerir continuamente, sem pausas entre as deglutições; $16,7 \%(\mathrm{n}=3)$ solicitaram ingerir o mais rápido possível. Além disso, um dos estudos $(5,5 \%)$ teve como orientação a ingestão continua ou da maneira habitual, e outro (5,5\%) a ingestão contínua e rápida. Em um estudo $(5,5 \%)$ essa informação não foi mencionada.

Os resultados quanto às caracterizações da deglutição sequencial são variados e descritos de acordo com os objetivos delineados em cada estudo. Visando permitir comparações, os estudos foram agrupados conforme seus achados.

Um estudo ${ }^{(23)}$ focou-se nas mudanças que ocorrem com a língua durante diferentes tipos de deglutição - única ou sequencial - em adultos saudáveis. Os autores concluíram que a língua tem um padrão de movimentação único, porém utiliza movimentos de propulsão com duração mais curta e com sobreposição de seus movimentos nos casos de deglutição sequencial, durante ingesta contínua de $200 \mathrm{ml}$ de água na borda do copo. Já um estudo comparando adultos e idosos saudáveis ${ }^{(21)}$ observou que com o envelhecimento há um aumento somente na duração do movimento da língua neste tipo de deglutição.

Dois outros estudos ${ }^{(4,22)}$ sobre a movimentação da língua em deglutições sequenciais observaram que, quando os sujeitos executam essas deglutições, os movimentos de língua são mais variáveis com o envelhecimento ${ }^{(4)}$ e há estabilidade da coordenação dos diferentes segmentos da língua com a mandíbula, diminuição na variabilidade dos movimentos de língua e mandíbula, além da sobreposição dos movimentos de língua ${ }^{(22)}$. Observou-se, ainda, maior variação no número de ciclos de movimentos verticais da língua para a deglutição, mesmo nos idosos mais jovens ${ }^{(4)}$.

A movimentação do complexo hiolaríngeo em deglutições sequenciais foi descrita por diferentes estudos ${ }^{(5,9,11,24,25)}$. Um estudo $^{(24)}$ encontrou a laringe elevada e o vestíbulo laríngeo fechado na maioria dos indivíduos. Outro estudo ${ }^{(5)}$ observou três tipos de padrão de movimentação do complexo hiolaríngeo: descida do complexo hiolaríngeo e abertura do vestíbulo laríngeo a cada deglutição em 53\% dos indivíduos, manutenção do fechamento do vestíbulo laríngeo e da elevação do complexo hiolaríngeo após cada deglutição em $27 \%$ e, por último, um tipo misto em $20 \%$ destes, alternando entre abertura e fechamento do vestíbulo laríngeo durante a sequência de deglutições com canudo. Outros estudos ${ }^{(9,11,25)}$ também observaram esses três tipos de movimentação com o canudo e/ou copo, porém com diferentes porcentagens.

Ainda em relação à movimentação laríngea, outro grupo de pesquisadores ${ }^{(26)}$ encontrou essencialmente dois padrões, sem diferença quanto à idade (adultos ou idosos) e ao gênero: $67,1 \%$ com descida do complexo hiolaríngeo e abertura do vestíbulo laríngeo a cada deglutição e $31,4 \%$ do tipo misto (alternando abertura e fechamento contínuo após cada deglutição). Comparando os estudos supracitados, vemos que o último ${ }^{(26)}$ tem um $n=70$, e sua comparação ao $n=15$ do estudo anterior ${ }^{(5)}$ pode explicar as diferenças encontradas entre os padrões de movimentação laríngea. Também devemos observar que um estudo $^{(26)}$ não utiliza canudo como utensílio, o que também pode influenciar nos achados.

Uma pesquisa ${ }^{(25)}$ foi conduzida com o objetivo de observar se a trajetória da movimentação hióidea influencia no grau de penetração laríngea durante a deglutição sequencial com canudo. Tal pesquisa reportou maior grau de penetração quando houve combinação entre a manutenção do fechamento do vestíbulo laríngeo e a elevação do complexo hiolaríngeo após cada deglutição, juntamente com um padrão de movimentação em que o hioide se move na direção ântero-superior após o início da deglutição, sem nenhum movimento além do plano mandibular.

Um estudo foi conduzido ${ }^{(9)}$ para averiguar se o movimento do complexo hiolaríngeo e a localização do bolo alimentar em pacientes com acidente vascular cerebral (AVC) agudo, sem disfagia significativa, e em adultos saudáveis afeta a segurança da deglutição sequencial. Observou-se ocorrência significativa de elevação parcial do complexo hiolaríngeo em adultos sadios e pós-AVC durante ingestão sequencial de $100 \mathrm{ml}$ de líquido. Em ambos os grupos, houve concordância quanto ao aumento da invasão de vias aéreas inferiores com deglutições sequenciais quando comparadas a deglutições únicas. Ambos os parâmetros não afetaram a segurança da deglutição. Tal estudo também observou que o fechamento laríngeo durante a deglutição sequencial é influenciado pelo utensílio e pela instrução sobre o modo de deglutir, o que não ocorre nas deglutições isoladas.

Um estudo ${ }^{(27)}$ observou que a entrada do bolo alimentar na faringe é comum durante uma refeição com velocidade normal em adultos saudáveis, com acúmulo em valéculas antes do início da deglutição. Essa entrada foi vista em $76 \%$ das deglutições de bolos sólidos e em $60 \%$ das deglutições de bolos líquidos (neste estudo, gerados pelo mascar de chicletes e não pela observação da ingestão de líquido durante uma refeição). Essa entrada do bolo na faringe esteve associada a uma breve adução das pregas vocais em ambos os casos. Verificou-se que o líquido tem trajetória distinta do sólido, dividindo-se e passando pelas paredes laterais em volta da laringe e se unindo novamente na hipofaringe.

Outro estudo ${ }^{(28)}$ também encontrou acúmulo de líquido na faringe antes da deglutição em adultos saudáveis. As autoras contestam o conceito difundido de atraso no trânsito faríngeo, pois, comparando deglutições únicas com deglutições sequenciais, há trânsito faríngeo mais lento no segundo caso, unicamente porque o bolo se localiza na hipofaringe antes do início da resposta faríngea. Em complementação, outro estudo ${ }^{(5)}$ afirma que a hipofaringe é um ponto de gatilho crucial para eliciar a deglutição faríngea quando há ingestão sequencial de líquido por canudo, em adultos jovens, diferentemente dos estudos com deglutições isoladas, em que a deglutição faríngea ocorre quando o bolo atinge os pilares anteriores das fauces. Comparando adultos e idosos saudáveis, um estudo ${ }^{(11)}$ não encontrou diferença com o avanço da idade, tendo sido a localização do bolo, inferior à valécula, mais encontrada que no nível da valécula ou superior a ela.

Em relação à invasão de vias aéreas na deglutição sequencial, $30 \%$ de adultos saudáveis apresentam pequenos episódios 
de penetração laríngea, com expulsão eficaz de seu conteúdo, ditos como parte de um comportamento normal nesse tipo de deglutição, segundo um estudo ${ }^{(28)}$. Outro trabalho ${ }^{(11)}$ verificou que grande parte dos idosos apresenta algum grau de invasão de vias aéreas, concluindo que com o avanço da idade, principalmente após os 70 anos, há uma alteração na habilidade de manter as vias aéreas protegidas, bem como de fazer o clareamento supraglótico.

Quanto à apneia da deglutição, um dos primeiros estu$\operatorname{dos}^{(12)}$, dentro do período estabelecido desta revisão, comparou adultos e idosos tanto saudáveis quanto pós-AVC. Os pesquisadores observaram que com o aumento do volume ingerido há um aumento no tempo de fechamento laríngeo e de abertura do cricofaríngeo, além da diminuição do contato da base da língua ${ }^{(12)}$.

Já outro grupo de pesquisadores ${ }^{(24)}$ se denomina o primeiro a estudar de maneira instrumental a apneia da deglutição e a fase da respiração a ela associada em adultos saudáveis, com volumes de água padronizados e com água no copo com canudo $(100 \mathrm{ml})$. Na pesquisa supracitada, não foi observada diferença significativa na duração dessa apneia com os três volumes padronizados, mas quando a água foi ofertada no canudo houve um prolongamento na duração da apneia. Os autores enfatizaram que a fase expiratória da respiração costuma ser relacionada à apneia da deglutição, porém com o canudo há variação nesse padrão, com alguns indivíduos inspirando após a deglutição. Também observaram, na ingestão da água com canudo, que a maioria dos indivíduos fez deglutições múltiplas de forma ininterrupta, ou seja, mantendo a apneia da deglutição. Para os autores, pessoas com doenças respiratórias estão expostas ao risco de aspiração ao tentar ingerir líquido com canudo com esse padrão de deglutições múltiplas.

Outro estudo ${ }^{(26)}$ também observou uma alta taxa de inspiração e abertura do vestíbulo laríngeo após a deglutição sequencial de líquido no copo, o que não ocorre na deglutição de pequeno volume de líquido. Para esses autores, a porcentagem maior de inspiração encontrada após a deglutição sugere que a deglutição sequencial na borda do copo pode colocar os sujeitos em risco de aspiração maior que as deglutições únicas, caso eles apresentem desordem da deglutição. Essa alta taxa de inspiração com deglutições sequenciais também foi observada por outro estudo ${ }^{(29)}$, não só no copo como também com o canudo. Já outra pesquisa comparando copo e canudo ${ }^{(30)}$ só obteve maior taxa de inspiração após a deglutição com o uso do canudo. Outro estudo ${ }^{(31)}$ afirma que o canudo aumenta a frequência das inspirações antes das deglutições sequenciais por demandar diminuição da pressão intraoral, que pode eliciar uma inspiração, além de causar desconforto respiratório em adultos saudáveis.

Dois estudos conduzidos pelo mesmo grupo de pesquisadores $^{(32,33)}$ avaliou a deglutição forçada com canudo, utilizando um volume de $200 \mathrm{ml}$. O primeiro estudo ${ }^{(32)}$ teve um grupo menor de participantes e observou que o volume do bolo e a pressão de sucção diminuem nos idosos com disfagia. Já o segundo ${ }^{(33)}$ constatou que idosos têm frequência elevada de deglutições múltiplas e maior frequência de inspiração após deglutição forçada no canudo.

\section{CONCLUSÃO}

O estudo da deglutição sequencial vem crescendo nos últimos anos, porém ainda não faz parte de boa parte dos protocolos de avaliação da deglutição.

Os principais achados atribuídos à deglutição sequencial em adultos e idosos são: variabilidade no fechamento do complexo hiolaríngeo; maior taxa de inspiração após a deglutição; entrada do bolo em faringe, com acúmulo ou não em valéculas antes da deglutição; presença de algum grau de penetração laríngea; prolongamento na duração da apneia da deglutição e sobreposição dos movimentos linguais.

Não foi encontrada padronização determinante de volume ofertado, de instrução dada e de exames utilizados para avaliar a deglutição sequencial. Estudos replicados tornam-se necessários para maior compreensão das características da deglutição sequencial em populações idosas e disfágicas.

Alguns achados não são comparáveis entre si, pelo número variável de participantes e pelo uso de diferentes utensílios. Assim, esta revisão apontou que não existe consenso sobre a influência do utensílio na deglutição sequencial. Considerando que a maioria dos estudos é realizada com sujeitos normais, vemos a dificuldade de extrapolar seus achados para os sujeitos com queixa de dificuldade de deglutição.

\section{REFERÊNCIAS}

1. Padovani AR, Moraes DP, Mangili LD, Andrade CRF. Protocolo fonoaudiológico de avaliação do risco para disfagia (PARD). Rev Soc Bras Fonoaudiol. 2007;12(3):199-205.

2. Sordi M, Mourão LF, Silva AA, Flosi LC. Importância da interdisciplinaridade na avaliação das disfagias: avaliação clínica e videofluoroscópica da deglutição. Braz J Otorhinolaryngol. 2009;75(6):776-87.

3. Santoro PP, Fúria CL, Forte AP, Lemos EM, Garcia RI, et al. Otolaryngology and speech therapy evaluation in the assessment of oropharyngeal dysphagia: a combined protocol proposal. Braz J Otorhinolaryngol. 2011;77(2):201-13.

4. Steele CM, Van Lieshout PH. Influence of bolus consistency on lingual behaviors in sequential swallowing. Dysphagia. 2004;19(3):192-206.

5. Daniels SK, Foundas AL. Swallowing physiology of sequential straw drinking. Dysphagia. 2001;16(3):176-82.

6. Bours GJ, Speyer R, Lemmens J, Limburg M, Wit R. Bedside screening tests vs. videofluoroscopy or fiberoptic endoscopic evaluation of swallowing to detect dysphagia in patients with neurological disorders: systematic review. J Adv Nurs. 2009;65(3):477-93.

7. Dziadziola J, Hamlet S, Michou G, Jones L. Multiple swallows and piecemeal deglutition; observations from normal adults and patients with head and neck cancer. Dysphagia. 1992;7(1):8-11.

8. Wu MC, Chang YC, Wang TG, Lin LC. Evaluating swallowing dysfunction using a $100-\mathrm{ml}$ water swallowing test. Dysphagia. 2004;19(1):43-7.

9. Murguia M, Corey DM, Daniels S. Comparison of sequential swallowing in patients with acute stroke and healthy adults. Arch Phys Med Rehabil. 2009;90(11):1860-5.

10. Bennett JW, Van Lieshout PH, Pelletier CA, Steele CM. Sip-sizing behaviors in natural drinking conditions compared to instructed experimental conditions. Dysphagia. 2009;24(2):152-8.

11. Daniels SK, Corey DM, Hadskey LD, Legendre C, Priestly DH, et al. Mechanism of sequential swallowing during straw drinking in healthy young and older adults. J Speech Lang Hear Res. 2004;47(1):33-45.

12. Lazarus CL, Logemann JA, Rademaker AW, Kahrilas PJ, Pajak T, et al. Effects of bolus volume, viscosity, and repeated swallows in nonstroke 
subjects and stroke patients. Arch Phys Med Rehabil. 1993;74(10):106670.

13. Lawless HT, Bender S, Oman C, Pelletier C. Gender, age, vessel size, cup vs. straw sipping, and sequence effects on sip volume. Dysphagia. 2003;18(3):196-202.

14. Leder SB, Suiter D, Green BG. Silent aspiration risk is volumedependent. Dysphagia. 2011;26(3):304-9.

15. Marques $\mathrm{CH}$, André $\mathrm{C}$, Rosso AL. Disfagia no AVE agudo: revisão sistemática sobre métodos de avaliação. Acta Fisiatr. 2008;15(2):106-10.

16. Souza MT, Silva MD, Carvalho RC. Revisão integrativa: o que é e como fazer. Einstein. 2010;8(1):102-6.

17. Lim SH, Lieu PK, Phua SY, Seshadri R, Venketasubramanian N, et al. Accuracy of bedside clinical methods compared with fiberoptic endoscopic examination of swallowing (FEES) in determining the risk of aspiration in acute stroke patients. Dysphagia. 2001;16(1):1-6.

18. Nathadwarawala KM, McGroary A, Wiles CM. Swallowing in neurological outpatients: use of a timed test. Dysphagia. 1994;9(2):120-9.

19. Hugues TA, Wiles CM. Clinical measurement of swallowing in health and in neurogenic dysphagia. QJM. 1996;89(2):109-16.

20. Hinds NP, Wiles CM. Assessment of swallowing and referral to speech and language therapists in acute stroke. QJM. 1998;91(12):829-35.

21. Steele CM, Van Lieshout PH. Tongue movements during water swallowing in healthy young and older adults. J Speech Lang Hear Res. 2009;52(5):1255-67.

22. Steele CM, Van Lieshout PH. The dynamics of lingual-mandibular coordination during liquid swallowing. Dysphagia. 2008;23(1):33-46.

23. Chi-Fishman G, Stone M, McCall GN. Lingual action in normal sequential swallowing. J Speech Lang Hear Res. 1998;41(4):771-85.
24. Martin BJ, Logemann JA, Shaker R, Dodds WJ. Coordination between respiration and swallowing: respiratory phase relationships and temporal integration. J Appl Physiol. 1994;76(2):714-23.

25. Tsushima C, Saitoh E, Baba M, Yokoyama M, Fujii W, et al. Hyoid movement and laryngeal penetration during sequential swallowing. J Med Dent Sci. 2009;56(3):113-21.

26. Dozier TS, Brodsky MB, Michel Y; Walters BC, Martin-Harris B. Coordination of swallowing and respiration in normal sequential cup swallows. Laryngoscope. 2006;116(8):1489-93.

27. Dua KS, Ren J, Bardan E, Xie P, Shaker R. Coordination of deglutitive glottal function and pharyngeal bolus transit during normal eating. Gastroenterology. 1997;112(1):73-83.

28. Chi-Fishman G, Sonies B. Motor strategy in rapid sequential swallowing: new insights. J Speech Lang Hear Res. 2000;43(6):1481-92.

29. Preiksaitis HG, Mills CA. Coordination of breathing and swallowing: effects of bolus consistency and presentation in normal adults. J Appl Physiol. 1996;81(4):1707-14.

30. Hirst LJ, Ford GA, Gibson GJ, Wilson JA. Swallow-induced alterations in breathing in normal older people. Dysphagia. 2002;17(2):152-61.

31. Lederle A, Hoit JD, Barkmeier-Kraemer J. Effects of sequential swallowing on drive to breathe in young, healthy adults. Dysphagia. 2012;27(2):221-7.

32. Nilsson H, Ekberg O, Olsson R, Hindfelt B. Oral function test for monitoring suction and swallowing in the neurologic patient. Dysphagia. 1995;10(2):93-100.

33. Nilsson H, Ekberg O, Olsson R, Hindfelt B. Quantitative aspects of swallowing in an elderly nondysphagic population. Dysphagia. 1996;11(3):180-4. 